\title{
Intrinsic leakage of the Josephson flux qubit and breakdown of the two-level approximation for strong driving
}

\author{
Alejandro Ferrón and Daniel Domínguez \\ Centro Atómico Bariloche and Instituto Balseiro, \\ 8400 San Carlos de Bariloche, Río Negro, Argentina.
}

\begin{abstract}
Solid state devices for quantum bit computation (qubits) are not perfect isolated two-level systems, since additional higher energy levels always exist. One example is the Josephson flux qubit, which consists on a mesoscopic SQUID loop with three Josephson junctions operated at or near a magnetic flux of half quantum. We study intrinsic leakage effects, i.e., direct transitions from the allowed qubit states to higher excited states of the system during the application of pulses for quantum computation operations. The system is started in the ground state and rf- magnetic field pulses are applied at the qubit resonant frequency with pulse intensity $f_{p}$. A perturbative calculation of the average leakage for small $f_{p}$ is performed for this case, obtaining that the leakage is quadratic in $f_{p}$, and that it depends mainly on the matrix elements of the supercurrent. Numerical simulations of the time dependent Schrödinger equation corresponding to the full Hamiltonian of this device were also performed. From the simulations we obtain the value of $f_{p}$ above which the two-level approximation breaks down, and we estimate the maximum Rabi frequency that can be achieved. We study the leakage as a function of the ratio $\alpha$ among the Josephson energies of the junctions of the device, obtaining the best value for minimum leakage $(\alpha \approx 0.85)$. The effects of flux noise on the leakage are also discussed.
\end{abstract}

PACS numbers: 03.67.Lx,82.25.Cp,74.50.+r

\section{INTRODUCTION}

In the last decade different devices of mesoscopic Josephson junctions have been studied experimentally as candidates for the design of quantum bits (qubits) $\underline{\underline{1}} \underline{\underline{10}}$ A large effort has been devoted to succeed in the coherent manipulation of their quantum states in a controllable way $1,3,4,6$ One of the superconductig qubit devices that has been studied in the last years is the Josephson flux qubit,,$\underline{2,6}$ which consists on a mesoscopic SQUID loop with three Josephson junctions operated at or near a magnetic flux of half quantum.

Real qubit devices, however, are not perfect isolated two-level systems. First, coupling to the external environment induces relaxation and dephasing,$\underline{5,8}$ Second, additional higher energy levels always exist in solid state devices. Therefore leakage effects, i.e., transitions from the allowed qubit states to higher excited states of the system can occur during quantum computation operations. ${ }^{11.12}$ Indirect leakage to the higher energy levels produced through the interaction with the environment has been studied in some cases $\stackrel{13,14}{\underline{1}}$ Even neglecting the interaction with the external environment, intrinsic leakage can occur due to direct transitions outside the computational subspace during the application of pulses for computational operations. $11,15,16$ Due to the importance of minimizing the gate errors due to leakage, several optimization strategies to compensate leakage, based on varying the pulse shapes and pulse sequences, have been studied recently: $\underline{17-21}$

Furthermore, the study of the multilevel dynamics of qubit devices has become of interest by itself in the last years $\underline{22-27}$ The consideration of the superconducting qubit devices as artificial atoms, has lead to the study of the dynamic effects of their level structure beyond the lowest energy levels. Effects of strong drive amplitudes on Rabi oscillations have been studied ${ }^{22-24}$ Driving the flux qubit with large amplitude harmonic excitations have also revealed the higher energy levels through Landau-Zener-Stuckelberg transitions ${ }^{25-27}$ Mach-Zender interferometry ${ }^{25,26}$ and amplitude spectroscopy ${ }^{27}$ have been the subject of recent studies of the flux qubit as an artificial atom. Moreover, it has also been pointed out that the high energy level structure of the Josephson flux qubit should show quantum signatures of classical chaos ${ }^{28}$

For quantum computation applications one wants to maximize the number of quantum bit operations before gate errors become important. In superconducting qubits, long pulses are limited by the decoherence due to the environment and short pulses by leakage out of the qubit computational subspace. In order to maximize the number of quantum bit operations, one has to maximize the ratio $t_{\mathrm{deph}} / t_{\mathrm{op}}$, where $t_{\mathrm{deph}}$ is the dephasing time and $t_{\mathrm{op}}$ is the time scale for a single quantum operation. The main approach has been to improve the design of the qubit devices to increase their coherence time. In the case of the Josephson flux qubit there has been an important progress in increasing $t_{\mathrm{deph}}$, from the early experiments by Chiorescu et al $\underline{\underline{6}}$ with $t_{\mathrm{deph}} \approx 20 \mathrm{~ns}$ to recent experiments that report $t_{\mathrm{deph}} \approx 0.5-2 \mu \mathrm{s} . \stackrel{8}{=}$ Provided that one has succeeded to achieve a $t_{\text {deph }}$ as large as possible for a given device, the following approach is to reduce $t_{\mathrm{op}}$. It is in this later case $\left(t_{\mathrm{op}} \ll t_{\mathrm{deph}}\right)$ when the effect of leakage is relevant. The usual strategy for quantum operations is to drive the qubit with a periodic pulse of intensity $f_{p}$ at a resonant frequency $\hbar \omega_{r}=E_{1}-E_{0}$, with $E_{1}-E_{0}$ the energy difference between the two qubit 
states. In this case, the time scale for quantum computing operations, $t_{o p}$, is proportional to the period of Rabi oscillations, $t_{o p} \sim T_{R}$. Since the time $T_{R}$ depends on the pulse strength as $T_{R} \sim 1 / f_{p}$, to reduce $t_{\mathrm{op}}$, one has to increase $f_{p}$. Along this line of reasoning, the following questions will be addressed here: (i) how much is possible to increase $f_{p}$ before leakage effects become important, and (ii) for which circuit parameters of the Josephson flux qubit the intrinsic leakage is minimum.

To this end, in this work we will study the quantum dynamics of the Josephson flux qubit solving its time dependent Schrödinger equation considering the full hamiltonian of the system. Since we are interested in time scales such that $t_{\text {op }} \ll t_{\text {deph }}$, the interaction with the environment will be neglected, and we focus on the calculation of the amount of intrinsic leakage. We will study the case when the qubit is driven by an rf pulse in the magnetic field that is resonant with $\hbar \omega_{r}=E_{1}-E_{0}$. The amount of leakage as a function of the pulse strength $f_{p}$ will be calculated both perturbatively for small $f_{p}$ and numerically for arbitrary values of $f_{p}$. The paper is organized as follows. In Sec.II we introduce the model hamiltonian and equations for the Josephson flux qubit. In Sec. III we present a perturbative calculation of the leakage in the case of an harmonic resonant drive. In Sec.IV we present our numerical results for the time dependent Schrödinger equation, calculating the amount of leakage as a function of $f_{p}$. In Sec.V we show results on the dependence of te leakage with different circuit parameters. In particuar, the optimum value of the circuit parameter $\alpha$ (the ratio among the Josephson couplings of the junctions of the qubit) will be computed. In Sec. VI we analyze the effect of an small amount of noise in the results shown in the previous section. Finally, Sec. VII contains a summary and a discussion of the most relevant points of our findings.

\section{MODEL FOR THE DEVICE FOR THE JOSEPHSON FLUX QUBIT}

The device used for the Josephson flux qubit ${ }^{2}$ consists on a superconducting ring with three Josephson junctions enclosing a magnetic flux $\Phi=f \Phi_{0}$, with $\Phi_{0}=h / 2 e$, see Fig 1.

The junctions have gauge invariant phase differences defined as $\varphi_{1}, \varphi_{2}$ and $\varphi_{3}$, respectively, with the sign convention corresponding to the directions indicated by the arrows in Fig 1. Typically the circuit inductance can be neglected and the phase difference of the third junction is: $\varphi_{3}=-\varphi_{1}+\varphi_{2}-2 \pi f$. Therefore the system can be described with two dynamical variables: $\varphi_{1}, \varphi_{2}$. The circuits that are used for the Josephson flux qubit have two of the junctions with the same coupling energy, $E_{J, 1}=E_{J, 2}=E_{J}$, and capacitance, $C_{1}=C_{2}=C$, while the third junction has smaller coupling $E_{J, 3}=\alpha E_{J}$ and capacitance $C_{3}=\alpha C$, with $0.5<\alpha<1$. The above

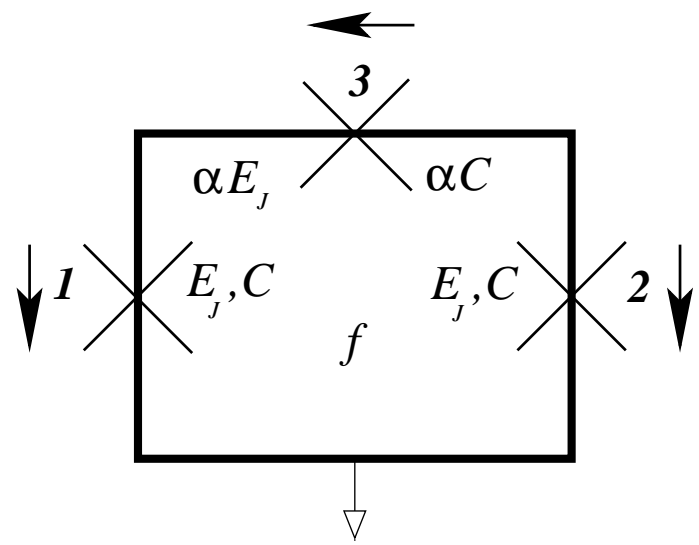

FIG. 1: Circuit for the Device for the Josephson Flux Qubit as described in the text. Josephson junctions 1 and 2 have Josephson energy $E_{J}$ and capacitance $C$, and junction 3 has Josephson energy and capacitance $\alpha$ times smaller. The arrows indicate the sign convention for defining the gauge invariant phase differences. The circuit encloses a magnetic flux $\Phi=f \Phi_{0}$.

considerations lead to the Hamiltonian ${ }^{2}$

$$
\mathcal{H}=\frac{1}{2} \vec{P}^{T} \mathbf{M}^{-1} \vec{P}+E_{J} V(\vec{\varphi}),
$$

where the two-dimensional coordinate is $\vec{\varphi}=\left(\varphi_{1}, \varphi_{2}\right)$. The potential energy term is given by the Josephson energy of the circuit and, in units of $E_{J}$, is:

$$
V(\vec{\varphi})=2+\alpha-\cos \varphi_{1}-\cos \varphi_{2}-\alpha \cos \left(2 \pi f+\varphi_{1}-\varphi_{2}\right) .
$$

The kinetic energy term is given by the electrostatic energy of the circuit, where the two-dimensional momentum is

$$
\vec{P}=\left(P_{1}, P_{2}\right)=\mathbf{M} \cdot \frac{d \vec{\varphi}}{d t},
$$

and $\mathbf{M}$ is an effective mass tensor determined by the capacitances of the circuit,

$$
\mathbf{M}=C\left(\frac{\Phi_{0}}{2 \pi}\right)^{2} \mathbf{m}
$$

with

$$
\mathbf{m}=\left(\begin{array}{cc}
1+\alpha & -\alpha \\
-\alpha & 1+\alpha
\end{array}\right)
$$

We neglected in $\mathbf{M}$ the on-site capacitances $C_{g}$ (typically $\left.C_{g} / C \sim 10^{-2}-10^{-3} \ll 1\right)$. The system modelled with Eqs. (11)-(2) is analogous to a particle with anysotropic mass $\mathbf{M}$ in a two-dimensional periodic potential $V(\vec{\varphi})$.

In typical junctions, the Josephson energy scale, $E_{J}$, is much larger than the electrostatic energy of electrons, $E_{C}=e^{2} / 2 C$, and the system is in a classical regime. On the other hand, mesoscopic junctions (with small area) 
can have $E_{J} \sim E_{C}$, and quantum fluctuations become important. In this case, the quantum momentum operator is defined as

$$
\vec{P} \rightarrow \hat{\vec{P}}=-i \hbar \nabla_{\varphi}=-i \hbar\left(\frac{\partial}{\partial \varphi_{1}}, \frac{\partial}{\partial \varphi_{2}}\right) .
$$

After replacing the above defined operator $\hat{\vec{P}}$ in the Hamiltonian of Eq.(1), the eigenvalue Schrödinger equation becomes

$$
\left[-\frac{\eta^{2}}{2} \nabla_{\varphi}^{T} \mathbf{m}^{-1} \nabla_{\varphi}+V(\vec{\varphi})\right] \Psi_{\nu}(\vec{\varphi})=E_{\nu} \Psi_{\nu}(\vec{\varphi}),
$$

where we normalized energy by $E_{J}$ and momentum by $\hbar / \sqrt{8 E_{C} / E_{J}}$. We defined in Eq.(3) the parameter $\eta=$ $\sqrt{8 E_{C} / E_{J}}$ which plays the role of an effective $\hbar \underline{\underline{28}}$ Typical flux qubit experiments have values of $\alpha$ in the range $0.6-0.9$ and $\eta$ in the range $0.1-0.6 \cdot \underline{\underline{6}} \underline{-10,27}$

In this work, we will study the quantum dynamics of the Josephson flux qubit. Therefore, we solve the timedependent Schrödinger equation which, with the same normalization as above, is given by

$$
i \frac{\partial \Psi(\vec{\varphi})}{\partial t}=\left[-\frac{\eta^{2}}{2} \nabla_{\varphi}^{T} \mathbf{m}^{-1} \nabla_{\varphi}+V(\vec{\varphi})\right] \Psi(\vec{\varphi}),
$$

where we normalized time by $t_{J}=\hbar / E_{J}$.

We integrate numerically Eq. (44) with a second order split-operator algorithm, $\stackrel{29}{=}$ using a discretization grid of $\Delta \varphi=2 \pi / 128$ and $\Delta t=0.1 t_{J}$. We use $2 \pi$-periodic boundary conditions on $\vec{\varphi}=\left(\varphi_{1}, \varphi_{2}\right)$. Eigenstates $\left|\Phi_{i}\right\rangle$ and eigenenergies $E_{i}$ are also calculated by numerical diagonalization of Eq. (3), with the same discretization grid and boundary conditions. In what follows we will consider the case of $\eta=0.48$ (i.e., $E_{J} / E_{C}=35$ ), which corresponds to the experiment of Chiorescu et al. $\underline{6}$

\section{PERTURBATIVE CALCULATION OF THE INTRINSIC LEAKAGE}

In quantum computation implementations $2,6,7$ the Josephson flux qubit is operated at magnetic fields near the half-flux quantum, $f=1 / 2+\delta f$, with $\delta f \ll 1$. For values of $\alpha \geq 1 / 2$, the potential of Eq.(2) has two well defined minima. At the optimal operation point $f_{0}=1 / 2$, the two lowest energy eigenstates $\left(\left|\Psi_{0}\right\rangle\right.$ and $\left.\left|\Psi_{1}\right\rangle\right)$ are symmetric and antisymmetric superpositions of two states corresponding to macroscopic persistent currents of opposite sign. A two-level truncation of the Hilbert space is usually performed $\stackrel{2}{2}$ In the subspace expanded by $\left|\Psi_{0}\right\rangle$ and $\left|\Psi_{1}\right\rangle$, the hamiltonian of Eq. (11) is reduced to

$$
\mathcal{H}=-\frac{\epsilon}{2} \hat{\sigma}_{z}-\frac{\Delta}{2} \hat{\sigma}_{x},
$$

where $\mathcal{H}$ is written in the qubit basis defined by $|0\rangle=$ $\left(\left|\Psi_{0}\right\rangle+\left|\Psi_{1}\right\rangle\right) / \sqrt{2}$ and $|1\rangle=\left(\left|\Psi_{0}\right\rangle-\left|\Psi_{1}\right\rangle\right) / \sqrt{2}$. Here
$\Delta=E_{1}-E_{0}$ is the two-level spliting at $f=1 / 2$, which increases strongly with $\alpha$, and $\epsilon=4 \pi \alpha E_{J} S_{01} \delta f$ (considering that $\delta f \ll 1)$, with $S_{01}=\left\langle\Psi_{0}\left|\sin \left(\pi+\varphi_{1}-\varphi_{2}\right)\right| \Psi_{1}\right\rangle$. (For typical values of $\alpha$ and $\eta$, one has $S_{01} \sim 0.8$ ). Most experiments control the system varying the magnetic field $\delta f=f-1 / 2$. Recently it has been shown experimentally that is also possible to manipulate the value of $\Delta$ by controlling $\alpha$, replacing the third junction by an additional SQUID loop $\underline{9,10}$

Most of the experiments on the flux qubit study the possibility of single bit quantum operations by driving the qubit with a resonant pulse in the magnetic flux with $\delta f(t)=f_{p} \sin \left(\omega_{r} t\right)$, at the resonant frequency $\hbar \omega_{r}=E_{1}-$ $E_{0}$. If the system is started in the ground state $\left|\Psi_{0}\right\rangle$, after the pulse is applied during a time interval $\tau$ the populations of the ground state and the excited state are

$$
\begin{aligned}
& P_{0}=\left|\left\langle\Psi(\tau) \mid \Psi_{0}\right\rangle\right|^{2}=\cos ^{2}\left(\Omega_{R} \tau / 2\right), \\
& P_{1}=\left|\left\langle\Psi(\tau) \mid \Psi_{1}\right\rangle\right|^{2}=\sin ^{2}\left(\Omega_{R} \tau / 2\right),
\end{aligned}
$$

with the Rabi frequency $\hbar \Omega_{R}=\epsilon_{p} / 2$ and $\epsilon_{p} \approx$ $4 \pi \alpha E_{J} S_{01} f_{p}$. This result is usually obtained in the rotating wave approximation (see below).

In order to check the goodness of the two-level approximation we are going to evaluate, perturbatively, the population of the higher energy levels when a pulse $\delta f(t)=f_{p} \sin (\omega t)$ is applied, with $f_{p} \ll 1$. We calculate the leakage outside of the quantum computational space spanned by the two lowest levels as

$$
\mathcal{L}(t)=\sum_{n=2}^{\infty}\left|\left\langle\Psi(t) \mid \Psi_{n}\right\rangle\right|^{2}=\sum_{n=2}^{\infty} P_{n}(t),
$$

where the $\left|\Psi_{n}\right\rangle$ are the eigenstates at $f_{0}=1 / 2$.

We now write the hamiltonian of Eq. (11) as $\mathcal{H}=$ $\mathcal{H}\left(f_{0}\right)+W(\delta f(t))$ with $f_{0}=1 / 2$ and

$$
\begin{aligned}
W(\delta f(t))= & \alpha E_{J} \sin [2 \pi \delta f(t)] \sin \left(\pi+\phi_{1}-\phi_{2}\right)+ \\
& 2 \alpha E_{J} \sin ^{2}[\pi \delta f(t)] \cos \left(\pi+\phi_{1}-\phi_{2}\right),
\end{aligned}
$$

for small $f_{p}$ we have

$$
W(\delta f(t)) \approx 2 \pi f_{p} \alpha E_{J} \sin (\omega t) \sin \left(\pi+\phi_{1}-\phi_{2}\right) .
$$

For the perturbative calculations we use the fact that the first and second states interact strongly with each other but only weakly with higher states. In this approximation we solve (see for example Ref 30 )

$$
\begin{aligned}
& i \hbar \frac{\partial c_{0}(t)}{\partial t}=W_{00}(t) c_{0}(t)+W_{01}(t) e^{i \omega_{01} t} c_{1}(t), \\
& i \hbar \frac{\partial c_{1}(t)}{\partial t}=W_{10}(t) e^{i \omega_{10} t} c_{0}(t)+W_{11}(t) c_{1}(t),
\end{aligned}
$$

$$
i \hbar \frac{\partial c_{n}(t)}{\partial t}=W_{n 0}(t) e^{i \omega_{n 0} t} c_{0}(t)+W_{n 1}(t) e^{i \omega_{n 1} t} c_{1}(t),
$$


with $W_{i j}=\left\langle\Psi_{i}|W| \Psi_{j}\right\rangle$ and $\omega_{i j}=\left(E_{i}-E_{j}\right) / \hbar$.

We rewrite equations (7) and (8) in the form

$$
\begin{gathered}
i \frac{\partial c_{0}(t)}{\partial t}=\Omega_{00} \sin (\omega t) c_{0}(t)+\Omega \sin (\omega t) e^{-i \omega_{10} t} c_{1}(t) \\
i \frac{\partial c_{1}(t)}{\partial t}=\Omega \sin (\omega t) e^{i \omega_{10} t} c_{0}(t)+\Omega_{11} \sin (\omega t) c_{1}(t),
\end{gathered}
$$

where we defined $\hbar \Omega_{i j}=2 \pi f_{p} \alpha S_{i j} E_{J}$ with $S_{i j}=$ $\left\langle\Psi_{i}\left|\sin \left(\pi+\phi_{1}-\phi_{2}\right)\right| \Psi_{j}\right\rangle$, and $\Omega_{10}=\Omega_{01}=\Omega$ (for $S_{i j}$ real). In order to solve these equations we make the following change of variables 31

$$
b_{j}(t)=c_{j}(t) e^{i\left(\Omega_{j j} / \omega\right) \cos (\omega t)} .
$$

then

$$
i \frac{\partial b_{0}(t)}{\partial t}=\Omega \sin (\omega t) e^{-i\left(\omega_{10} t+\nu \cos (\omega t)\right)} b_{1}(t)
$$

and

$$
i \frac{\partial b_{1}(t)}{\partial t}=\Omega \sin (\omega t) e^{i\left(\omega_{10} t+\nu \cos (\omega t)\right)} b_{0}(t)
$$

where $\nu=\left(\Omega_{11}-\Omega_{00}\right) / \omega$. Using the relation

$$
e^{i x \cos \theta}=\sum_{k=-\infty}^{\infty} i^{k} J_{k}(x) e^{i n \theta},
$$

we obtain

$$
\begin{array}{r}
i \frac{\partial b_{0}(t)}{\partial t}=\frac{\Omega}{2 i} \sum_{k} i^{k} J_{k}(-\nu)\left[e^{-i\left(\omega_{10}-(k+1) \omega\right) t}\right. \\
\left.-e^{-i\left(\omega_{10}-(k-1) \omega\right) t}\right] b_{1}(t), \\
i \frac{\partial b_{1}(t)}{\partial t}=\frac{\Omega}{2 i} \sum_{k} i^{k} J_{k}(\nu)\left[e^{i\left(\omega_{10}+(k+1) \omega\right) t}\right. \\
\left.-e^{i\left(\omega_{10}+(k-1) \omega\right) t}\right] b_{1}(t),
\end{array}
$$

where the $J_{k}$ are Bessel functions. When $\omega \simeq \omega_{10}$ we can use the rotating wave approximation (RWA) ${ }^{31,32}$ and negelect the highly off resonant terms obtaining:

$$
\frac{\partial b_{0}(t)}{\partial t}=-\gamma e^{-i\left(\omega_{10}-\omega\right) t} b_{1}(t)
$$

and

$$
\frac{\partial b_{1}(t)}{\partial t}=\gamma e^{i\left(\omega_{10}-\omega\right) t} b_{0}(t)
$$

where $\gamma=(\Omega / 2)\left(J_{0}(\nu)+J_{2}(\nu)\right)$. In the exact resonance case $\left(\omega=\omega_{10}\right)$ the problem has analytic solution.

$$
\frac{\partial b_{0}(t)}{\partial t}=-\gamma b_{1}(t) ; \quad \frac{\partial b_{1}(t)}{\partial t}=\gamma b_{0}(t) .
$$

If the system was initially in the ground state, we obtain

$$
c_{0}(t)=e^{i\left(\Omega_{00} / \omega\right)(1-\cos (\omega t))} \cos (\gamma t)
$$

and

$$
c_{1}(t)=e^{i\left(\Omega_{00} / \omega-\left(\Omega_{11} / \omega\right) \cos (\omega t)\right)} \sin (\gamma t) .
$$

We see that $\left|c_{0}(t)\right|^{2}=\cos ^{2}(\gamma t)$ and $\left|c_{1}(t)\right|^{2}=\sin ^{2}(\gamma t)$, which allows to identify the Rabi frequency as $\Omega_{R}=2 \gamma$, and therefore:

$$
\begin{aligned}
\Omega_{R} & =\Omega\left[J_{0}(\nu)+J_{2}(\nu)\right] \\
& =\frac{2 \pi f_{p} \alpha S_{01} E_{J}}{\hbar}\left[J_{0}(\nu)+J_{2}(\nu)\right],
\end{aligned}
$$

with $\nu=\frac{2 \pi f_{p} \alpha E_{J}}{\hbar \omega}\left(S_{11}-S_{00}\right)$.

Now we must solve (9) using the two-level solutions of Eqs.(19) and (20).

$i \frac{\partial c_{n}(t)}{\partial t}=\Omega_{n 0} \sin (\omega t) e^{i \omega_{n 0} t} c_{0}(t)+\Omega_{n 1} \sin (\omega t) e^{i \omega_{n 1} t} c_{1}(t)$

it is easy to show, using (19) and (20), that

$$
\begin{aligned}
\frac{\partial c_{n}(t)}{\partial t}= & e^{i \Omega_{00} / \omega} \sum_{k=-\infty}^{\infty} i^{k+1}\left[i \beta _ { n k 0 } \left(e^{i a_{n k 0}^{+} t}+e^{i b_{n k 0}^{+} t}\right.\right. \\
& \left.-e^{i a_{n k 0}^{-} t}-e^{i b_{n k 0}^{-} t}\right)+\beta_{n k 1}\left(e^{i a_{n k 1}^{+} t}+e^{i b_{n k 1}^{-} t}\right. \\
& \left.\left.-e^{i a_{n k 1}^{-} t}-e^{i b_{n k 1}^{+} t}\right)\right]
\end{aligned}
$$

where $a_{n k i}^{ \pm}=k \omega+\omega_{n i} \pm \omega+\gamma, b_{n k i}^{ \pm}=k \omega+\omega_{n i} \pm \omega-$ $\gamma$ and $\beta_{n k i}=\left(\Omega_{n i} / 4\right) J_{k}\left(-\Omega_{i i} / \omega\right)$. We then obtain an expression for the coefficients

$$
\begin{aligned}
c_{n}(t)= & e^{i \Omega_{00} / \omega} \sum_{k=-\infty}^{\infty} e^{i(k+1) \pi / 2}\left[\beta _ { n k 0 } \left(\frac{e^{i a_{n k 0}^{+} t}}{a_{n k 0}^{+}}+\frac{e^{i b_{n k 0}^{+} t}}{b_{n k 0}^{+}}\right.\right. \\
& \left.-\frac{e^{i a_{n k 0}^{-} t}}{a_{n k 0}^{-}}-\frac{e^{i b_{n k 0}^{-} t}}{b_{n k 0}^{-}}\right)+\beta_{n k 1}\left(\frac{e^{i a_{n k 1}^{+} t}}{a_{n k 1}^{+}}+\frac{e^{i b_{n k 1}^{-} t}}{b_{n k 1}^{-}}-\right. \\
& \left.\left.\frac{e^{i a_{n k 1}^{-} t}}{a_{n k 1}^{-}}-\frac{e^{i b_{n k 1}^{+} t}}{b_{n k 1}^{+}}\right)\right]+\delta_{n k 0}-i \delta_{n k 1},
\end{aligned}
$$

where $\delta_{n k 0}=\beta_{n k 0}\left(1 / a_{n k 0}^{-}+1 / b_{n k 0}^{+}-1 / a_{n k 0}^{+}-1 / b_{n k 0}^{-}\right)$ and $\delta_{n k 1}=\beta_{n k 1}\left(1 / a_{n k 1}^{+}+1 / b_{n k 1}^{-}-1 / a_{n k 1}^{-}-1 / b_{n k 1}^{+}\right)$.

The average leakage leakage out of the subspace spanned by the first two levels is: 


$$
\overline{\mathcal{L}}=\sum_{n=2}^{\infty} \overline{\left|c_{n}(t)\right|^{2}}
$$

where $\overline{\left|c_{n}(t)\right|^{2}}$ means a time average of $\left|c_{n}(t)\right|^{2}$. From (24) we obtain the perturbative result for the average leakage as:

$$
\begin{array}{r}
\overline{\mathcal{L}}=\sum_{n=2}^{\infty} \sum_{k=-\infty}^{\infty} \beta_{n k 0}^{2}\left(\frac{1}{\left(a_{n k 0}^{+}\right)^{2}}+\frac{1}{\left(b_{n k 0}^{+}\right)^{2}}\right. \\
\left.+\frac{1}{\left(a_{n k 0}^{-}\right)^{2}}+\frac{1}{\left(b_{n k 0}^{-}\right)^{2}}\right)+\beta_{n k 1}^{2}\left(\frac{1}{\left(a_{n k 1}^{+}\right)^{2}}+\frac{1}{\left(b_{n k 1}^{-}\right)^{2}}+\right. \\
\left.\frac{1}{\left(a_{n k 1}^{-}\right)^{2}}+\frac{1}{\left(b_{n k 1}^{+}\right)^{2}}\right) \\
+\left(\sum_{k=-\infty}^{\infty} \delta_{n k 0} \cos (k \pi / 2)+\delta_{n k 1} \sin (k \pi / 2)\right)^{2} \\
+\left(\sum_{k=-\infty}^{\infty} \delta_{n k 0} \sin (k \pi / 2)-\delta_{n k 1} \cos (k \pi / 2)\right)^{2} .
\end{array}
$$

We can simplify the final expression for the leakage taking into account that $\Omega_{i i} \ll \omega$, since the diagonal matrix elements are $\left|S_{i i}\right| \ll 1$. In this case the term $k=0$ of the Bessel functions is dominant in the expansion. We obtain

$$
\overline{\left|c_{n}(t)\right|^{2}} \approx \frac{\Omega_{n 0}^{2}}{\epsilon_{n 0}^{2}}+\frac{\Omega_{n 1}^{2}}{\epsilon_{n 1}^{2}},
$$

with

$$
\frac{1}{\epsilon_{n i}^{2}}=\frac{J_{0}^{2}\left(\Omega_{i i} / \omega\right)}{16}\left(z_{n i}+y_{n i}\right)
$$

and

$$
\begin{gathered}
y_{n i}=\left(\frac{1}{\omega_{n i}-\omega+\gamma}+\frac{1}{\omega_{n i}+\omega-\gamma}\right. \\
\left.-\frac{1}{\omega_{n i}+\omega+\gamma}-\frac{1}{\omega_{n i}-\omega-\gamma}\right)^{2}, \\
z_{n i}=\frac{1}{\left(\omega_{n i}-\omega+\gamma\right)^{2}}+\frac{1}{\left(\omega_{n i}+\omega-\gamma\right)^{2}}+ \\
\frac{1}{\left(\omega_{n i}+\omega+\gamma\right)^{2}}+\frac{1}{\left(\omega_{n i}-\omega-\gamma\right)^{2}} .
\end{gathered}
$$

Using that $\hbar \Omega_{n i}=2 \pi f_{p} \alpha S_{n i}$, with $S_{n i}=\left\langle\Phi_{n}\right| \sin (\pi+$ $\left.\phi_{1}-\phi_{2}\right)\left|\Phi_{0}\right\rangle$, we finally obtain:

$$
\overline{\mathcal{L}}=\frac{4 \pi^{2} f_{p}^{2} \alpha^{2}}{\hbar^{2}} \sum_{n=2}^{\infty}\left(\frac{\left|S_{n 0}\right|^{2}}{\epsilon_{n 0}^{2}}+\frac{\left|S_{n 1}\right|^{2}}{\epsilon_{n 1}^{2}}\right)
$$

\section{STRONG DRIVING AND BREAKDOWN OF THE TWO-LEVEL APPROXIMATION}

In this section we solve numerically the time-dependent Schrödinger equation of the full hamiltonian for the Josephson flux qubit, as given by Eq. (4). As a function of time we calcultate the population of the different energy eigenstates when a resonant pulse is applied. In this way we can evaluate directly how large is the population of the two lowest energy levels and the amount of leakage as a function of the pulse strength.

We first solve numerically the eigenvalue equation of Eq. (31) for $f_{0}=0.5$, obtaining the eigenvectors $\left|\Psi_{i}\right\rangle$ and eigenvalues $E_{i}$. Then we solve the corresponding timedependent Shcrödinger equation of Eq. (44).

We start the time evolution at the ground state $\left|\Psi_{0}\right\rangle$ for $f_{0}=0.5$. We apply a pulse in the magnetic field, $f(t)=f_{0}+\delta f(t)$, during a time interval $\tau$. After the pulse is applied, the wave function has evolved to $|\Psi(\tau)\rangle$ and the qubit returns to $f=f_{0}=0.5$. We calculate the population $P_{i}$ of the different eigenstates $\left|\Psi_{i}\right\rangle$, obtained at the end of the pulse: $P_{i}(\tau)=\left|\left\langle\Psi(\tau) \mid \Phi_{i}\right\rangle\right|^{2}$. The leakage outside of the quantum computational subspace expanded by the two lowest eigenstates, $\left|\Psi_{0}\right\rangle,\left|\Psi_{1}\right\rangle$, is then obtained as

$$
\mathcal{L}(\tau)=1-P_{0}(\tau)-P_{1}(\tau)=\sum_{i=2}^{\infty} P_{i}(\tau) .
$$

We consider a resonant rf field pulse, $\delta f(t)=$ $f_{p} \sin \left(\omega_{r} t\right)$, for $0<t<\tau$, with the resonant frequency $\hbar \omega_{r}=E_{1}-E_{0}$. In the experimental measurements of Rabi oscillations of Ref 6 , pulses of intensity in the range $5 \times 10^{-5} \lesssim f_{p} \lesssim 5 \times 10^{-4}$ were used, as it can be deduced from their data. Here we have calculated the time evolution of the Schrödinger equation in a wider range of parameters for the pulse strengths: $10^{-5}<f_{p}<0.1$.

In this section of the work we are going to present results for a typical experimental configuration that corresponds to the work of Chiorescu et al. $\underline{\underline{6}}$. In Fig.2 we show $\mathcal{L}(\tau)$ as a function of the pulse length $\tau$ for three different cases of $f_{p}$ for $\alpha=0.8$. We see that $\mathcal{L}(\tau)$ has strong oscil-

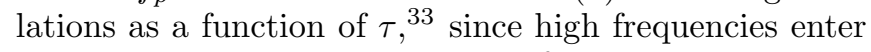
into place due to the contribution of several energy levels. At $f_{p} \sim 10^{-4}$, a typical value for experiments, the average value of the leakage is very small, $\mathcal{L} \sim 10^{-7}$, showing that under a resonant pulse the Josephson flux qubit behaves very closely as a two-level system. In constrast, a non-resonant pulse can have a higher leakage for similar pulse strengths, as it has been shown in Ref 16 for a constant dc pulse. In our results in Fig.2, we see that for increasing values of $f_{p}$ the leakage $\mathcal{L}(\tau)$ increases, reaching values of $10^{-3}$ for $f_{p} \sim 0.01$. A low frequency modulation can be clearly observed in Fig.2(a). This corresponds to the Rabi frequency of the approximate two-level system. The two-level Rabi frequency increases with $f_{p}$. Indeed, a "Rabi modulation" of the leakage can also be seen in Fig.2(b) at a higher frequency than in Fig.2(a). On the 

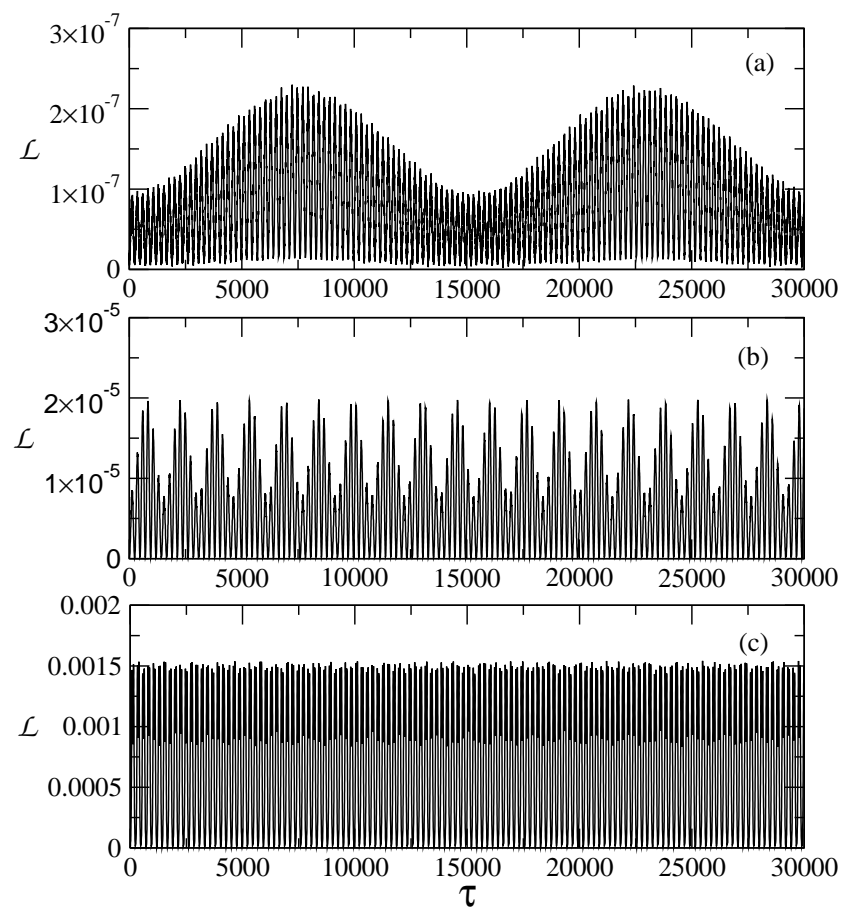

FIG. 2: Leakage $\mathcal{L}$ as a function of the pulse length $\tau$, for an rf field pulse of strength $f_{p}$ at the resonant frequency $\hbar \omega_{r}=$ $E_{1}-E_{0}$. (a) $f_{p}=0.0001$; (b) $f_{p}=0.001$; (c) $f_{p}=0.01$. Time is normalized by $t_{J}=\hbar / E_{J} \sim 0.5 \mathrm{ps}$.

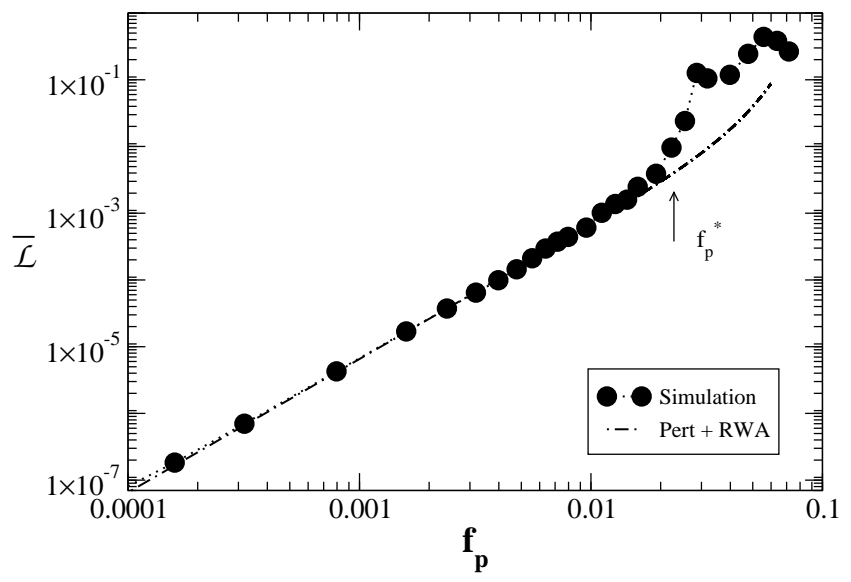

FIG. 3: Filled circles: Time averaged leakage $\overline{\mathcal{L}}$ as a function of the pulse strength $f_{p}$ for $\alpha=0.8$ for an $\mathrm{rf}$ field pulses of strength $f_{p}$ at the resonant frequency $\hbar \omega_{r}=E_{1}-E_{0}$. Dashdotte line: perturbative approximation of Eq.(26).

other hand, for $f_{p}=0.01$ in Fig.2(c) the expected Rabi frequency is high enough that it can not be distinguished from the other high frequencies contributing to the leakage. As we will see below, for $f_{p}$ above this case the two-level approximation is no longer adequate.

To evaluate more quantitatively the effect of strong pulses in the amount of leakage, we calculate the time
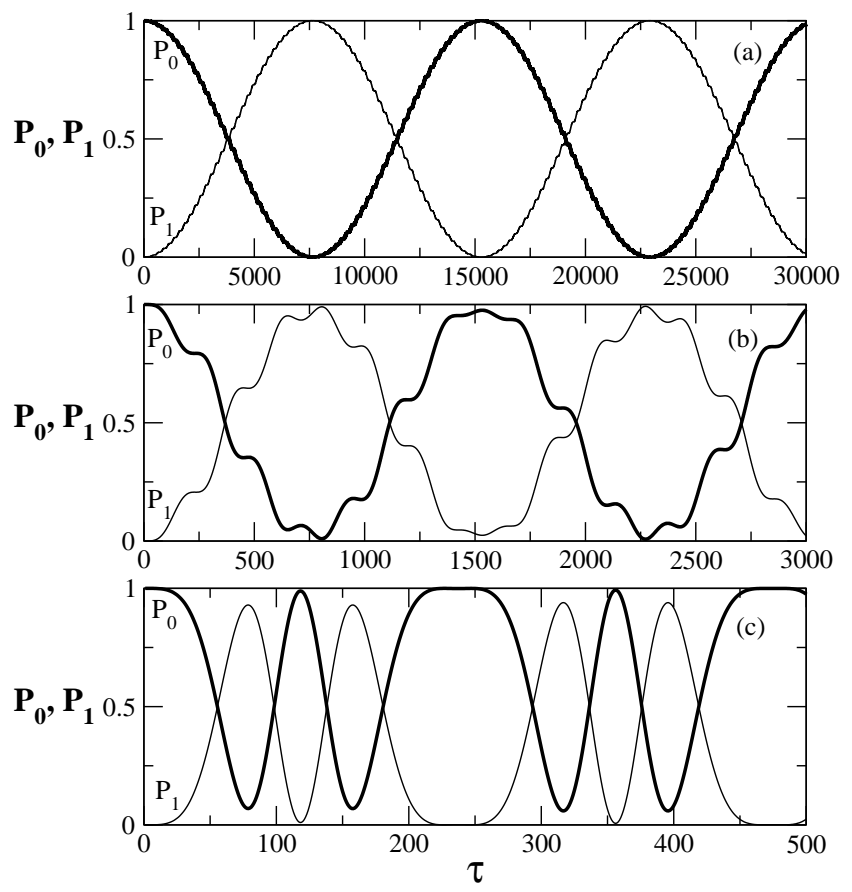

FIG. 4: Populations of the ground state, $P_{0}(\tau)$ (thick line), and the first excited state, $P_{1}(\tau)$ (thin line) as a function of the pulse length $\tau$, for an rf field pulse of strength $f_{p}$ at the resonant frequency $\hbar \omega_{r}=E_{1}-E_{0}$. (a) $f_{p}=0.0001$; (b) $f_{p}=0.001 ;$ (c) $f_{p}=0.01$. Time is normalized by $t_{J}=\hbar / E_{J}$.

averaged leakage $\overline{\mathcal{L}}$ as a function of the pulse intensity $f_{p}$. We show this result in Fig.3 for $\alpha=0.8$. We observe that $\overline{\mathcal{L}}$ grows quadratically with $f_{p}$ for low pulse strengths. At large values of $f_{p}$ the dependence of $\overline{\mathcal{L}}$ with $f_{p}$ clearly departs from this behavior. We compare in Fig.3 the numerical results with the perturbative approximation of Eq.(26) (summing up to the first 10 levels). We find that for $f_{p} \lesssim f_{p}^{*}=0.02$ the perturbative approximation is very good. For higher values of the pulse strength, the Eq.(26) no longer describes the behavior of $\overline{\mathcal{L}}\left(f_{p}\right)$, and the the average leakage increases quickly with $f_{p}$. In particular we find that for $f_{p} \sim 0.03$ the amount of leakage is important (i.e., near 10\%). From these results, we conclude that the two-level approximation can not be a good description of the dynamics for pulse strengths $f_{p}>f_{p}^{*}$.

We show in Fig.4 the population of the ground state $P_{0}(\tau)$ and the first excited state $P_{1}(\tau)$, for the same values of $f_{p}$ as in Fig.2. In Fig.4(a), for $f_{p}=0.0001$, we see a clear Rabi oscillation of the populations of the two levels of the qubit. A small modulation at high frequencies is also observed which is due to the perturbation of the higher energy levels. In Fig.4(b), for $f_{p}=0.001$, the perturbation of the high energy levels is more important, but Rabi oscillations can still be distinguished. In Fig.4(c), for $f_{p}=0.01$, we see that the behavior of $P_{0}(\tau)$ and $P_{1}(\tau)$ departs clearly from the simple Rabi oscillation scheme and a more complex oscillation with 


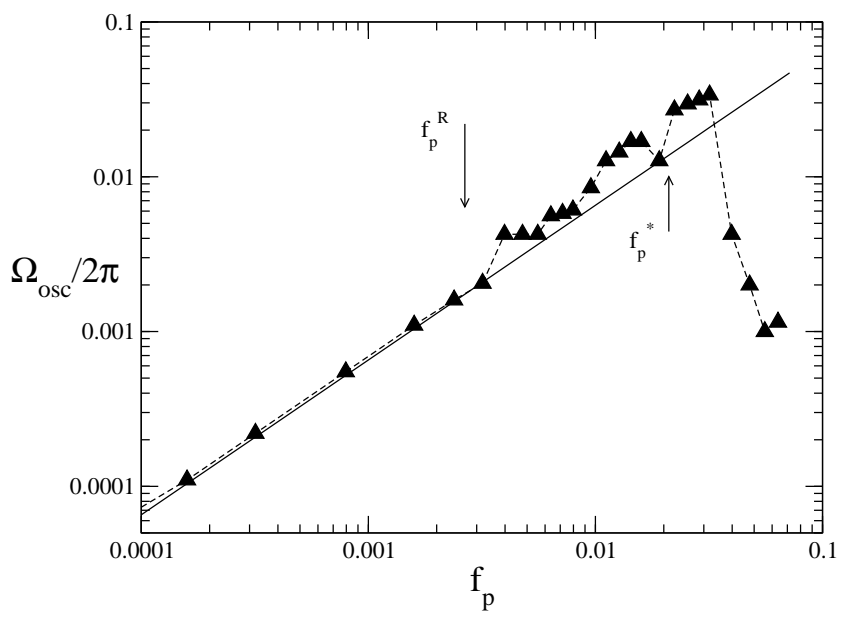

FIG. 5: Triangles: Main oscillation frequency $\Omega_{\mathrm{osc}}$ as a function of the pulse strength $f_{p}$ for $\alpha=0.8$. Line: Rabi frequency $\Omega_{R}$ as obtained in the rotating-wave approximation, Eq. (21)

two competing frequencies is observed. In this case the quantum operation of the qubit, if intended, will be more complex since it needs to be based in the knowledge of the oscillation pattern.

We now analize the Fourier power spectrum of the populations $P_{0}(\tau)$ and $P_{1}(\tau)$. For example for $P_{0}(\tau)$ we calculate $\hat{P}(\omega)=1 / T\left|\int_{0}^{T} \exp (i \omega t) P_{0}(t) d t\right|^{2}$. We define the dominant frequency of the oscillation from the maximum of the power spectrum, $\Omega_{\mathrm{osc}}=\max _{\omega} \hat{P}(\omega)$. A plot of the obtained $\Omega_{\text {osc }}$ as a function of the pulse strength $f_{p}$ is shown in Fig.5. We also plot the Rabi frequency $\Omega_{R}$ given by Eq.(21), which was obtained in the rotating-wave approximation. We observe that $\Omega_{\mathrm{osc}}$ is linear with $f_{p}$ in good agreement with Eq.(21), it i.e. $\Omega_{\mathrm{osc}} \approx \Omega_{R}$ for $f_{p} \lesssim f_{p}^{R}=0.003$. For larger values of $f_{p}, \Omega_{\text {osc }}$ departs from the linear dependence of $\Omega_{R}$. The rotating wave approximation that lead to Eq.(21) is valid if $\Omega_{R}<\omega_{r}=\left(E_{1}-E_{0}\right) / \hbar$. Indeed, $\Omega_{\text {osc }}$ reaches this value at $f_{p}^{R}$ (we see that for $f_{p}=f_{p}^{R}=0.003$ we have $\Omega_{\text {osc }} / 2 \pi \approx 0.002$, while $\left.\left(E_{1}-E_{0}\right) / 2 \pi=0.002107\right)$. Therefore, for $f_{p}>f_{p}^{R}$ we do not expect to find simple sinusoidal Rabi oscillations. Instead, more complex oscillations are observed, as seen in Fig.4(c).

From the above analysis we conclude that for $f_{p} \gtrsim$ $f_{p}^{R}=0.003$ the possibility of use of the device for quantum operations becomes more difficult due to the lack of Rabi oscillations. This implies that the highest possible Rabi frequency that can be obtained is, for $\alpha=0.8$,

$$
\Omega_{R}^{\max } / 2 \pi \approx 0.003 \alpha E_{J} / \hbar \approx 5 \mathrm{GHz}
$$

for $E_{J} \approx(2 \pi \hbar) 300 \mathrm{GHz}$, and using $\Omega_{R} \approx 2 \pi \alpha f_{p} E_{J}$. In the range $f_{p}^{*}>f_{p}>f_{p}^{R}$, the two level approximation is still a good approximation, since the average leakage is relatively small, but its use for quantum operations would not be as simple as in the case of $f_{p}<f_{p}^{R}$. For pulse strengths $f_{p}>f_{p}^{*}$ the intrinsic leakage is very important
$(\mathcal{L} \sim 0.1)$ and the device can not be treated as a two-level system. Moreover, we see in Fig.5 that at $f_{p} \sim f_{p}^{*}$, the dependence of the frequency $\Omega_{\text {osc }}$ with $f_{p}$ has a drastic change, very far apart from a "Rabi regime".

\section{DEPENDENCE OF THE INTRINSIC LEAKAGE WITH CIRCUIT PARAMETERS}

In this section of the paper we are going to study the behavior of the leakage as a function of the different parameters that define the circuit of the flux qubit. A simple argument is that leakage effects should be small if the energy difference between the third and the second level is much larger than the energy difference between the two lowest levels, i.e. if $E_{2}-E_{1} \gg E_{1}-E_{0}$. While this is generally correct, the magnitude of the matrix elements for the transition rates to higher energy levels can be even more important, as we will show in this section. Indeed, from the perturbative calculation of Eq.(26) we see that, besides an overall factor proportional to $f_{p}^{2} \alpha^{2}$, the average leakage $\overline{\mathcal{L}}$ depends both on the matrix elements $S_{n i}$, and the factors $\epsilon_{n i}$ (which are basically dominated by the energy level differences $E_{n}-E_{i}$ ).

Among the circuit parameters that can be varied either by external sources or by circuit design are $f_{0}, \alpha$ and $E_{J} / E_{C}$. As we mentioned before the qubit is operated at a dc magnetic field near the half-flux quantum, with $f_{0} \simeq$ $1 / 2$. In order to see what happens when we move out of the symmetry point $f_{0}=1 / 2$ we plot in Fig.6(a) the time averaged leakage as a function of $f_{0}$ for a fixed value of $f_{p}$. We can see from this figure that the leakage grows as we move appart of the symmetry point. This issue can be understood with Fig.6(b). Here we can see that if we move out of the symmetry point the distance between the two lower levels, $\Delta_{10}=E_{1}-E_{0}$ grows while the third level becomes closer to the second one, and thus $\Delta_{21}=$ $E_{2}-E_{1}$ decreases. This basically explains the increase of the leakage when moving out of the symmetry point $f_{0}=1 / 2$. Further information can be obtained from Fig.6(c) where we show the matrix element $\left|S_{12}\right|^{2}$, which corresponds to the supercurrent $\sin \left(\phi_{1}-\phi_{2}-2 \pi f\right)$ taken between the first excited level and the second excited level. We note that it has a minimum at $f_{0}=0.498$. The existence of this minimum correlates with the fact that the dependence of the leakage with $f_{0}$ has a shoulder near this point.

In principle, the amount of leakage will depend also on the $\alpha$-parameter since this parameter controls the shape of the effective potential in the hamiltonian, in particular the heigth of the barrier between the two potential minima. We have calculated numerically the average leakage $\overline{\mathcal{L}}$ as a function of $\alpha$ for a fixed pulse strengths $f_{p}$ at $f_{0}=1 / 2$. This is shown in Fig.7(a). We see that $\overline{\mathcal{L}}$ tends to decrease with increasing $\alpha$, contrary to what the $\alpha^{2}$ factor of Eq.(26) would have suggested. Moreover, we see that there is a minimum value for the leakage at $\alpha_{\min } \approx 0.85$. Therefore, there is an optimum value of the 

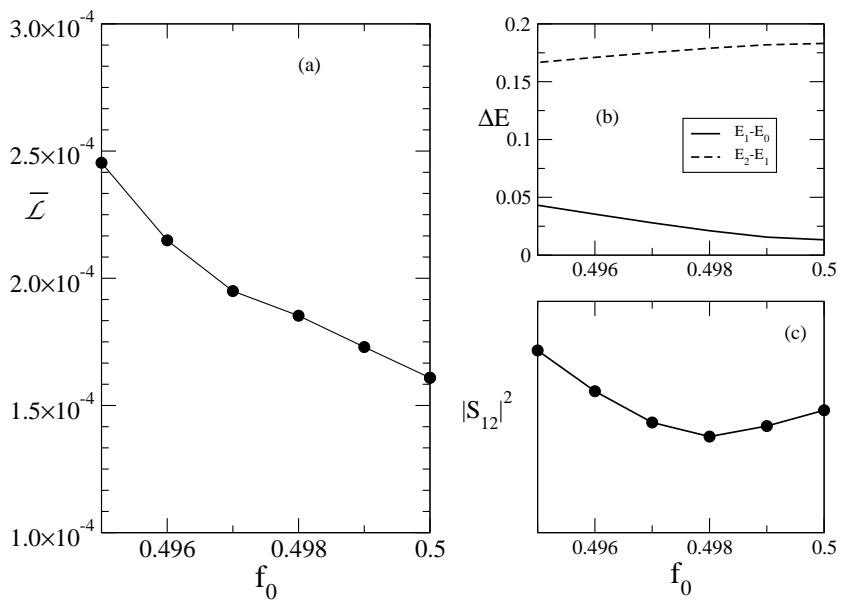

FIG. 6: (a) Time averaged leakage $\overline{\mathcal{L}}$ as a function of $f_{0}$ for fixed amplitude $f_{p}, E_{J} / E_{C}=35$ and $\alpha=0.8$. (b) Distance between the lower energy levels as a function of $f_{0}$. (c) Matrix element $\left|S_{12}\right|^{2}$ as a function of $f_{0}$.
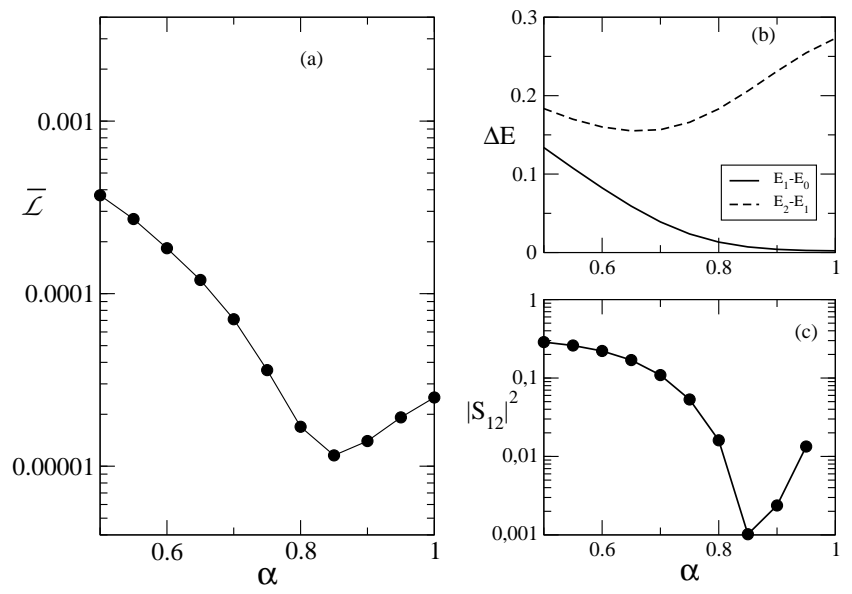

FIG. 7: (a) Time averaged leakage $\overline{\mathcal{L}}$ as a function of $\alpha$ for fixed amplitude $f_{p}, E_{J} / E_{C}=35$ and $f_{0}=0.5$. (b) Distance between the lower energy levels as a function of $\alpha$. (c) Matrix element $\left|S_{12}\right|^{2}$ as a function of $\alpha$.

circuit parameter $\alpha$ for which the leakage will be mimimum and the two-level approximation more adequate.

We plot in Fig.7(b) the difference between the three lowest energy levels as a function of $\alpha$ and in Fig.7(c) we plot the matrix element $\left|S_{12}\right|^{2}$ vs. $\alpha$. The energy distance $\Delta_{10}$ strongly decreases with $\alpha$, while $\Delta_{21}$ has a smooth non-monotonous dependence with $\alpha$. This later result, where $\Delta_{10} / \Delta_{21}$ decreases with $\alpha$ contributes to the general tendency of the leakage to decrease with increasing $\alpha$. However, the important result here is that the minimum leakage at $\alpha_{\min } \approx 0.85$ is directly correlated with a minimum in the dependence of $\left|S_{12}\right|^{2}$ with $\alpha$, as it can be observed in Fig.7(c). Therefore, it is this matrix element, for transitions between the second and

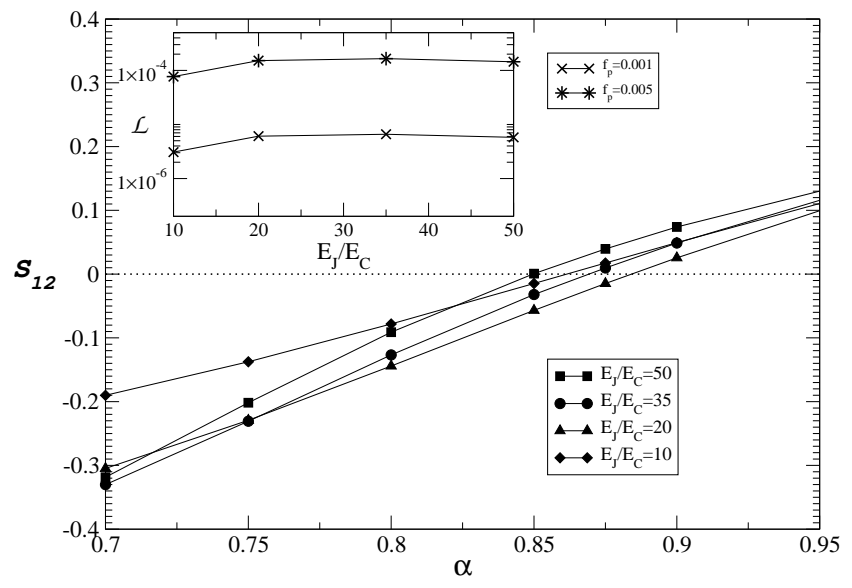

FIG. 8: Matrix element $S_{12}$ as a function of $\alpha$ for different values of $E_{J} / E_{C}$. The inset shows the time averaged leakage as a function of $E_{J} / E_{C}$ for two different values of the amplitude.

the third level, the factor that dominates the dependence of the leakage with $\alpha$.

In Fig.8 we show the amplitude of the matrix element $S_{12}$ as a function of $\alpha$ for different values of $E_{J} / E_{C}$. We observe that the minimum for $\left|S_{12}\right|^{2}$ shown in Fig.7(c) actually corresponds to the fact that $S_{12}$ crosses zero and therefore it vanishes at a particular value of $\alpha$. Furthermore, we find that for all the cases of $E_{J} / E_{C}$ analyzed, the matrix element becomes zero within the range of $0.83<\alpha<0.89$, nearly the same value of $\alpha$. In the inset in Fig. 8 we show how the amount of leakage (for a fixed value of $f_{p}, \alpha$, etc) changes with the ratio $E_{J} / E_{C}$. We see that $\overline{\mathcal{L}}$ does not change significantly, showing a slight increase for increasing $E_{J} / E_{C}$. This can be understood in the sense the dynamics of the system becomes more "classical" when increasing $E_{J} / E_{C}$, (the effective $\hbar$ decreases), the energy level structure becomes more crowded, and thus the effect of higher energy levels will tend to be more relevant.

\section{EFFECTS OF WEAK NOISE ON THE INTRINSIC LEAKAGE}

The results of the previous sections have been obtained in the ideal case in which the effect of the environment is neglected. The aim of this section is to analize how the small perturbation of a weak noise can affect the calculations of the intrinsic leakage of the previous sections. In superconducting qubits various sources of relaxation and decoherence are present due to the environment. Recent experiments in the Josephson flux qubit have shown that the dominant source of decoherence is due to $1 / f$ noise of the magnetic flux in the SQUID loop. $\underline{\underline{8}}$ Here we will make the simplification of treating the noise in the magnetic flux as a classical noise. In fact, it is usually 


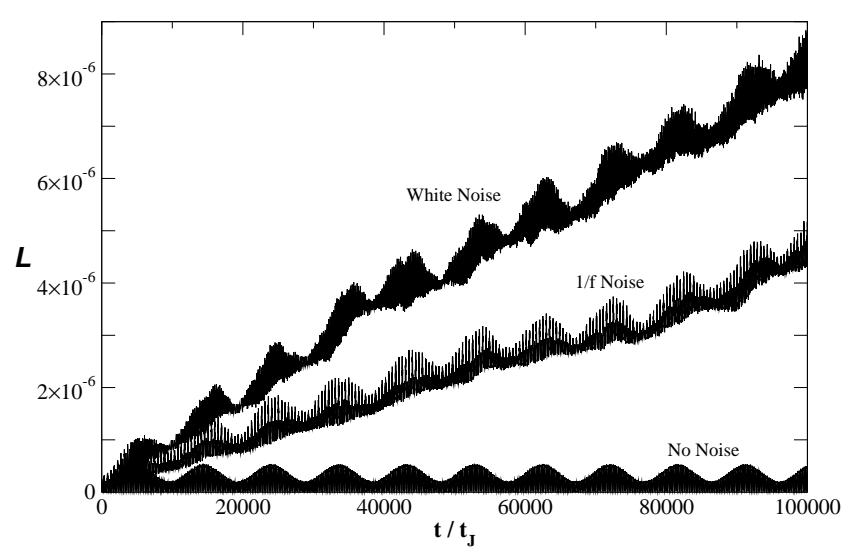

FIG. 9: Leakage $\mathcal{L}$ as a function of the pulse length $\tau$, for an $\mathrm{rf}$ field pulse of strength $f_{\mathrm{p}}=0.00016$ at the resonant frequency $\hbar \omega_{r}=E_{1}-E_{0}$ in the presence of white noise of intensity $A_{w}=7.10^{-6}$ and $1 / f$ noise with 250 bistable fluctuators and intensity $A_{f}=5 \cdot 10^{-5}$. Time is normalized by $t_{J}=\hbar / E_{J} \sim$ 0.5 ps.

assumed that for time scales much smaller than the energy relaxation time, the initial decoherence of the qubit can be described with a classical noise $\underline{\underline{34}}$ Thus, in the presence of an rf-drive, we take the magnetic flux as

$$
f(t)=\frac{1}{2}+f_{p} \sin (\omega t)+\delta f_{n}(t),
$$

Here, $\delta f_{n}(t)$ is the noise source in the magnetic flux, with average $\left\langle\delta f_{n}(t)\right\rangle=0$ and correlations $\left\langle\delta f_{n}(t) \delta f_{n}\left(t^{\prime}\right)\right\rangle=$ $A^{2} g\left(t-t^{\prime}\right)$.

We now solve numerically the time dependent Schrödinger equation, Eq.(44), when the rf-pulse is applied under the presence of noise, as given by Eq.(27). We calculate the leakage as in the previous section, but now averaging over 50 realizations of the noise. In Fig 9 we show the leakage $\mathcal{L}(\tau)$, obtained numerically, as a function of the pulse length, for a small value of $f_{p}$, with Gaussian white noise and with $1 / f$ noise. In the first case, we have $g\left(t-t^{\prime}\right)=\delta\left(t-t^{\prime}\right)$, and the calculations were done for white noise intensity $A_{w} \sim 10^{-5}$. In the second case the $1 / f$ noise is defined as the sum of several bistable fluctuators as studied, for example, in Ref. 34 . Here we considered 250 bistable fluctuators with intensity $A_{f} \sim 10^{-5}$. As a comparison, in the experiments the flux noise intensity is estimated to be of the order of $A \sim 10^{-6}$ at frequencies of $1 \mathrm{~Hz}, \underline{8}$ In the figure we can see that, besides the oscillations, there is a general linear increase of the leakage as $\mathcal{L}(t) \approx \Gamma_{L} t$, which probably implies an exponential dependence at long times as $\mathcal{L}(t) \approx 1-\exp \left(-\Gamma_{L} t\right)$. As we can see in this figure the case of $1 / f$ noise and white noise show quite similar behavior in their functional form. (The intensities of the two noises shown in Fig 9 have been chosen such that they result in different leak rates $\Gamma_{L}$ and thus they can be distinguished in the plot). The expected difference of the realistic case of $1 / f$ noise with a short-time correlated noise could be in the functional form of the dependence of the leakage $\mathcal{L}(t)$ for large times (larger than the time scale used in the present calculation).

We can calculate analytically an approximate result for the leakage if we assume that the noise intensity $A$ is small and that the noise is short-time correlated within a small time scale $\tau_{n}$, such that $A^{2} t_{J} \ll \tau_{n} \ll T_{R}$, with $T_{R}$ the period of the Rabi oscillations. In Section III we solved the two level system without noise in the RWA approximation. Here, we add the effect of noise as a perturbation within the RWA approximation. We now write the time dependent perturbation term of the Hamiltonian, $W(\delta f(t))$, for small values of $f_{p}$ and small noise intensity, as

$$
W(\delta f(t)) \approx 2 \pi \alpha \sin \left(\pi+\phi_{1}-\phi_{2}\right)\left(f_{p} \sin (\omega t)+\delta f_{n}(t)\right) .
$$

We then solve equation (9) with $W_{n i}(t)=$ $2 \pi \alpha S_{n i} E_{J}\left(f_{p} \sin (\omega t)+\delta f_{n}(t)\right)$ with the coefficients obtained from the two level approximation with noise (equations (7) and (8)). Integrating equation (9) and keeping only the terms with $A^{2}$ and $f_{p}^{2}$ we obtain

$$
\begin{aligned}
\left|c_{n}(t)\right|^{2}= & \left|c_{n}^{(0)}(t)\right|^{2}+\frac{(2 \pi \alpha A)^{2}}{\hbar^{2} \gamma}\{ \\
& \left.S_{n 0} S_{n 1} J_{0}\left(\Omega_{00} / \omega\right) J_{0}\left(\Omega_{11} / \omega\right) P(0) \sin ^{2}(\gamma t)\right) \\
& +\frac{\left|S_{n 0}\right|^{2} J_{0}^{2}\left(\Omega_{00} / \omega\right)}{4}[\gamma(P(\gamma)+P(-\gamma)) t \\
& +P(0) \sin (2 \gamma t))]+ \\
& \frac{\left|S_{n 1}\right|^{2} J_{0}^{2}\left(\Omega_{11} / \omega\right)}{4}[\gamma(P(\gamma)+P(-\gamma)) t \\
& -P(0) \sin (2 \gamma t)]\}
\end{aligned}
$$

where $c_{n}^{(0)}(t)$ is the coefficient obtained in absence of noise in equation (24), and $P(\omega)=\int_{-\infty}^{\infty} e^{i \omega t} g(t) d t$ is the noise spectral density. We see in Eq.(28) that, besides the oscillating terms, there are terms that contribute with an increase of $\left|c_{n}(t)\right|^{2}$ which is linear in $t$. This will give an overall increase of the leakage as $\mathcal{L}(t)=\sum_{n=2}^{\infty}\left|c_{n}(t)\right|^{2} \approx \Gamma_{L} t \approx 1-\exp \left(-\Gamma_{L} t\right)$, which defines $\Gamma_{L}$ as the leak rate. Typically, in the calculation of relaxation and dephasing, a $1 / f$ noise gives a Gaussian decay law insted of an exponential decay $\stackrel{8}{-}$ Similarly, one could expect that the exponential dependence of the leakage in the presence of short-time correlated noise could be modified to a different time dependence in the case of $1 / f$ noise (possibly a quadratic law), but as we showed above, such a difference is not distinguished in our numerical calculations.

Then, under the assumption of small short-time correlated noise, we obtain the leak rate as

$$
\Gamma_{L}=\frac{(2 \pi \alpha A)^{2}}{2 \hbar^{2}}\left[P\left(\frac{\Omega_{R}}{2}\right)+P\left(\frac{-\Omega_{R}}{2}\right)\right] \times
$$




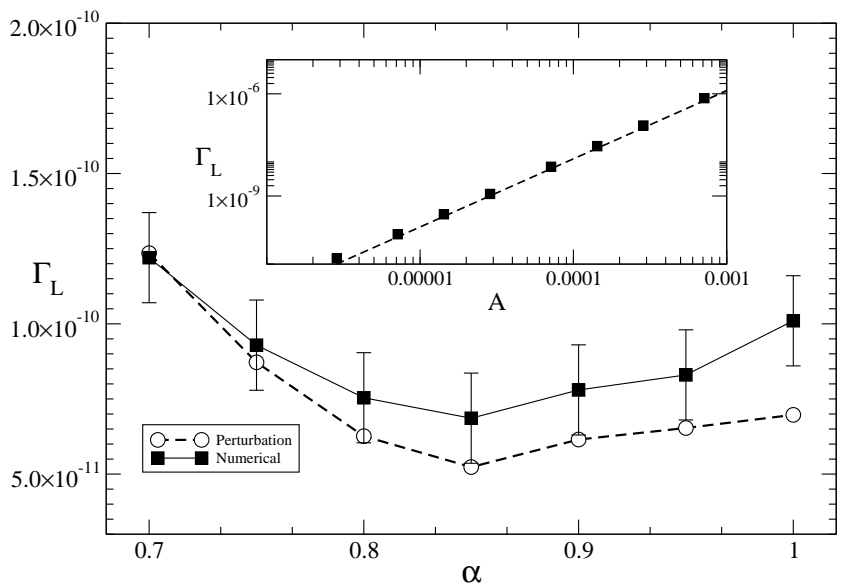

FIG. 10: Leak rate $\Gamma_{L}$ as a function of $\alpha$ for $f_{p}=0.00016$ and white noise strength $A=7 \times 10^{-6}$. Squares: numerical result. Circles: perturbative calculation. Inset: Leak rate $\Gamma_{L}$ as a function of the white noise strength $A$ for $\alpha=0.8$ and $f_{p}=0.00016$. Squares: numerical results. Dashed line: perturbative calculation.

$$
\left[\sum_{n=2}^{\infty}\left|S_{n 0}\right|^{2} J_{0}^{2}\left(\Omega_{00} / \omega\right)+\left|S_{n 1}\right|^{2} J_{0}^{2}\left(\Omega_{11} / \omega\right)\right] .
$$

From the numerical results for the white noise, we can make a linear fit of the time dependence of $\mathcal{L}(t)$ to obtain an estimate of the leak rate $\Gamma_{L}$. In the inset of Fig[10 we show the numerically obtained $\Gamma_{L}$ as a function of the white noise intensity $A$. We compare this result with the perturbative calculation of Eq.(29). As we can see, the agreement is excelent.

Finally, for a small value of the white noise intensity $A$, we plot the leak rate $\Gamma_{L}$ as a function of the circuit parameter $\alpha$. As we can see in Fig. 10 the leak rate has a minimum near $\alpha=0.85$. Therefore we observe that the optimum value of $\alpha$ for minimum leakage remains the same when a small white noise is added in the system. We also compare the numerical result with the perturbative calculation of Eq.(29). It shows an overall agreement with the existence of a minimum for $\alpha=0.85$. We find a small systematic difference for $\alpha>0.8$. This can be attributed to the numerical fit procedure used for estimating $\Gamma_{L}$, which may need the consideration of larger time intervals to improve the reliability of the fit.

\section{SUMMARY AND DISCUSSION}

We have presented numerical and perturbative calculations of the intrinsic leakage in the Josephson flux qubit when the system is driven by a rf resonant pulse. As a function of the pulse strength $f_{p}$ three regimes have been found: (i) For $f_{p}<f_{p}^{R}$, the leakage is very small $\mathcal{L} \ll 0.001$ and the device shows good Rabi oscillations. This is the regime in which the device can be operated as a qubit in a simple way. The perturbative calculations based on the rotating-wave approximation reproduce very well the numerical results in this case. (ii) For $f_{p}^{R}<f_{p}<f_{p}^{*}$, the leakage is still small, $\mathcal{L}<0.01$, and the device responds as a two-level system, but the response is more complex than simple sinusoidal Rabi oscillations. (iii) For $f_{p}>f_{p}^{*}$, the leakage is important, $\mathcal{L} \sim 0.1$ and the two-level approximation breaks down. We also find that the maximum Rabi frequency that can be achieved with this device, for $f_{p}=f_{p}^{R}$, is about $\Omega_{R} / 2 \pi \approx 5 \mathrm{GHz}$. This is a factor of 5-10 times larger than what is usually achieved in typical experiments, meaning that in principle is possible to use stronger pulses and correspondingly to further reduce the qubit operation time $t_{\mathrm{op}}$.

These results refer to the intrinsic leakage, i.e. the leakage due to direct transitions to the higher energy levels, and therefore the effect of the environment has been neglected. The interaction with the environment also adds further leakage effects due to indirect transitions to higher energy levels, see for example Ref 13.14. Concerning this point, in Sec. VI we have shown that a weak perturbation of the external world, considered as a small classical noise, does not change the qualitative dependence with circuit parameters of the leakage obtained in the previous sections for the ideal case. These results should be valid as soon as the Rabi period is much smaller than the decoherence time, which is the situation analyzed in this paper.

Here we have considered sharp pulses. Several strategies to reduce leakage varying the pulse shape and the type of pulse sequences have been discussed recently. $17-21$ Applying these strategies the value of the leakage could be further reduced from the values obtained here. According to our results, the use of these strategies will be of interest in the regime of $f_{p}<f_{p}^{R}$, when simple Rabi oscillations can be observed in the Josephson flux qubit.

One importan result of this work is the dependence of the leakage with the $\alpha$ parameter of the device. We have found that there is an optimum value near $\alpha \approx 0.85$ for minimum leakage. This result remains valid when the effect of noise of small amplitude in the magnetic flux is considered. We have found in this case that the magnitude of the leakage is dominated by the matrix element of the supercurrent for transitions between the first and the second excited level. When the amplitude of this matrix element crosses zero, we find that the leakage is minimum. Moreover, the Fig.7 shows that small values of $\alpha$ give large leakage, while the range $0.75 \lesssim \alpha<1$ is more favourable. Of course, the choice of $\alpha$ in the fabrication of the device should take into account other factors as dependence with $\alpha$ of the gap, the relaxation rate, the decoherence time, etc. Interestingly, the experiment of Ref 9 shows that the relaxation rate is stronger for small values of $\alpha$, which goes in the same direction as what we have observed here for the leakage.

Recently, Lucero et al. ${ }^{21}$ have used a procedure (called "Ramsey filtering") to measure the population of the second excited level in the superconducting phase qubit. It 
will be interesting if a similar procedure could be implemented in the Josephson flux qubit. In particular, it will be possible to measure experimentally the dependence of the leakage with $\alpha$ by using "Ramsey filtering" in the recently developed circuit for tuning the gap (i.e. $\alpha$ ) of the superconducting flux qubit. 9,10

The results obtained here show quantitatively how the multilevel dynamics can become relevant for strong driving amplitudes in the flux qubit, going beyond the twolevel approximation. Indeed, the recent experiments on amplitude spectroscopy of Ref 27 show how the use of strong ac drivings can be turned as a tool to reveal the energy level structure of the flux qubit device, now studied as a solid-sate artificial atom.

\section{Acknowledgments}

We acknowledge financial support from ANPCyT (PICT-2007-00824.), CNEA and Conicet.
1 Y. Nakamura, Y. A. Paskin, J. S. Tsai, Nature 398, 786 (1999).

2 J. E. Mooij, T.P.Orlando, L.S. Levitov, L. Tian, C.H. van der Wal and S. Lloyd, Science 285, 1036 (1999); T.P.Orlando, J.E. Mooij, L. Tian, C.H. van der Wal, L.S. Levitov, S. Lloyd, J.J. Mazo, Phys. Rev. B 60, 15398 (1999).

3 D. Vion, A. Aassime, A. Cottet, P. Joyez, H. Pothier, C. Urbina, D. Esteve, and M. H. Devoret, Science 296, 886 (2002).

4 J. M. Martinis, S. Nam, J. Aumentado and C. Urbina, Phys. Rev. Lett. 89, 117901 (2002); Y. Yu, Y. Yu, S. Han, X. Chu, S.-I. Chu, and Z. Wang, Science 296, 889 (2002).

5 Y. Makhlin, G. Schön, and A. Shnirman, Rev. Mod. Phys. 73, 357 (2001).

${ }^{6}$ I. Chiorescu, Y. Nakamura, C. J. P. M. Harmans, and J. E. Mooij, Science 299, 1869 (2003).

7 I. Chiorescu, P. Bertet, K. Semba, Y. Nakamura, C. J. P. M. Harmans, and J. E. Mooij, Nature 431, 159 (2004); E. Il'ichev, N. Oukhanski, A. Izmalkov, Th. Wagner, M. Grajcar, H.-G. Meyer, A. Yu. Smirnov, A. Maassen van den Brink, M. H. S. Amin, and A. M. Zagoskin, Phys. Rev. Lett. 91, 097906 (2003); Y. Yu, D. Nakada, J. C. Lee, B. Singh, D. S. Crankshaw, T. P. Orlando, W. D. Oliver, and K. K. Berggren, Phys. Rev. Lett. 92, 117904 (2004); A. Lupascu, C. J. M. Verwijs, R. N. Schouten, C. J. P. M. Harmans, and J. E. Mooij, Phys. Rev. Lett. 93, 177006 (2004); P. Bertet, I. Chiorescu, G. Burkard, K. Semba, C. J. P. M. Harmans, D. P. DiVincenzo, and J. E. Mooij, Phys. Rev. Lett. 95, 257002 (2005).

8 F. Yoshihara et al., Phys. Rev. Lett 97, 167001 (2006); K. Kakuyanagi et al., Phys. Rev. Lett. 98, 047004 (2007).

9 F. G. Paauw, A. Fedorov, C. J. Harmans, and J. E. Mooij Phys. Rev. Lett. 102, 090501 (2009)

10 Y. Shimazu, Y. Saito, and Z. Wada J. Phys. Soc. Japan 78, 064708 (2009)

11 R. Fazio et al., Phys. Rev. Lett. 83, 5385 (1999).

12 L. Tian and S. LLoyd, Phys. Rev. A, 62, 050301 (2000)

13 G. Burkard, R. H. Koch, and D. P. DiVincenzo, Phys. Rev. B 69, 064503 (2004).

14 F. Meier and D. Loss, Phys. Rev. B 71, 094519 (2005); T. Hakioglu and K. Savran, Phys. Rev. B 71, 115115 (2005).

15 X. Hu and S. Das Sarma, Phys. Rev. A 66, 012312 (2002); F. Troiani et al., Phys. Rev. Lett. 94, 207208 (2005).

16 D. Domínguez and E. N. Pozzo, J. Phys.: Conf. Series 150, 052045 (2009).

17 L.-A.Wu, M. S. Byrd, and D. A. Lidar, Phys. Rev. Lett. 89, 127901 (2002); M. S. Byrd et al., Phys. Rev. A 71,
$052301(2005)$.

18 Matthias Steffen, John M. Martinis, and Isaac L. Chuang, Phys. Rev. B 68, 224518 (2003).

19 Z. Zhou et al., Phys. Rev. Lett. 95, 120501 (2005).

20 P. Rebentrost, and F. K. Wilhelm, Phys. Rev. B 79, 060507 (2009); F. Motzoi, J. M. Gambetta, P. Rebentrost, F. K. Wilhelm, Phys. Rev. Lett. 103, 110501 (2009).

21 Erik Lucero, M. Hofheinz, M. Ansmann, Radoslaw C. Bialczak, N. Katz, Matthew Neeley, A. D. O'Connell, H. Wang, A. N. Cleland, and John M. Martinis, Phys. Rev. Lett. 100, 247001 (2008)

22 J. Claudon, F. Balestro, F. W. Hekking, and O. Buisson Phys. Rev. Lett. 93, 187003 (2004) J. Claudon, A. Fay, E. Hoskinson, and O. Buisson Phys. Rev. B 76, 024508 (2007) J. Claudon, A. Zazunov, F. W. J. Hekking, and O. Buisson Phys. Rev. B 78, 184503 (2008).

23 Yang Yu, D. Nakada, J. C. Lee, B. Singh, D. S. Crankshaw, T. P. Orlando, K. K. Berggren, and W. D. Oliver, Phys. Rev. Lett. 92, 117904 (2004) Z. Dutton, K. V. R. M. Murali, W. D. Oliver, and T. P. Orlando, Phys. Rev. B 73, 104516 (2006).

${ }^{24}$ S. K. Dutta, Frederick W. Strauch, R. M. Lewis, Kaushik Mitra, Hanhee Paik, T. A. Palomaki, Eite Tiesinga, J. R. Anderson, Alex J. Dragt, C. J. Lobb, and F. C. Wellstood Phys. Rev. B 78, 104510 (2008).

${ }^{25}$ W. D. Oliver, Y. Yu, J. C. Lee, K. K. Berggren, L. S. Levitov, and T. P. Orlando, Science 310, 1653 (2005); D. M. Berns, W. D. Oliver, S. O. Valenzuela, A. V. Shytov, K. K. Berggren, L. S. Levitov, and T. P. Orlando, Phys. Rev. Lett. 97, 150502 (2006).

26 A. Izmalkov, M. Grajcar, E. Il'ichev, N. Oukhanski, Th. Wagner, H.-G. Meyer, W. Krech, M. H. S. Amin, A. Maassen van den Brink, and A. M. Zagoskin, Europhys. Lett. 65, 844 (2004).

27 D. M. Berns, M. S. Rudner, S. O. Valenzuela, K. K. Berggren, W. D. Oliver, L. S. Levitov, and T. P. Orlando, Nature 455, 51 (2008); M. S. Rudner, A. V. Shytov, L. S. Levitov, D. M. Berns, W. D. Oliver, S. O. Valenzuela, and T. P. Orlando, Phys. Rev. Lett. 101, 190502 (2008); W D. Oliver and S. O. Valenzuela, Quantum Inf. Process 8, 261 (2009).

28 E. N. Pozzo and D. Domínguez, Phys. Rev. Lett. 98, 057006 (2007); E. N. Pozzo, D. Domínguez, and M. J. Sánchez, Phys. Rev. B 77, 024518 (2008).

29 M. D. Feit et al., J. Comp. Phys. 47, 412 (1982).

30 E. Merzbacher, Quantum Mechanics, 2nd edition, John Wiley \& Sons, 1970, p. 454.

31 N. Aravantinos-Zafiris and Emmanuel Paspalakis, Phys. 
Rev. A 72, 014303 (2005).

32 A. Brown, W. J. Meath and P. Tran, Phys. Rev. A 63, 013403 (2000).

${ }^{33}$ In Fig. 2 the intrinsic leakage comes back to the same value almost periodically in time because we are consider the dynamics in the ideal case, neglecting the effect of the environment. The solution of the time dependent Schrodinger equation always gives almost periodic oscillations of the populations of the eigenstates. At longer time scales, the effect of the environment will start to be important, and then the leakage $\mathcal{L}(\tau)$ should increase with time. This effect is taken into account later in Fig. 9 in Sec. VI, in an approximate semiclassical treatment of noise effects.

${ }^{34}$ G. Falci, A. D'Arrigo, A. Mastellone, and E. Paladino, Phys. Rev. Lett. 94, 167002 (2005). 